\title{
Topology-Optimized 4D Printing of a Soft Actuator
}

\author{
Ali Zolfagharian ${ }^{1}$, Martin Denk ${ }^{2}$, Mahdi Bodaghi ${ }^{3}$, Abbas Z. Kouzani ${ }^{1}$, Akif Kaynak ${ }^{1}$ \\ ${ }^{1}$ School of Engineering, Deakin University, Geelong, 3216, Australia \\ ${ }^{2}$ Munich University of Applied Sciences, Munich, 80335, Germany \\ ${ }^{3}$ Department of Engineering, School of Science and Technology, Nottingham Trent \\ University, Nottingham, NG11 8NS, United Kingdom
}

\begin{abstract}
Soft robots and actuators are emerging devices providing more capabilities in the field of robotics. More flexibility and compliance attributing to soft functional materials used in the fabrication of these devices make them ideal for delivering delicate tasks in fragile environments, such as food and biomedical sectors. Yet, the intuitive nonlinearity of soft functional materials and their anisotropic actuation in compliant mechanisms constitute an existent challenge in improving their performance. Topology optimization (TO) along with four-dimensional (4D) printing is a powerful digital tool that can be used to obtain optimal internal architectures for the efficient performance of porous soft actuators. This paper employs TO analysis for achieving high bending deflection of a 3D printed polyelectrolyte actuator, which shows bending deformations in response to electrical stimuli in an electrolyte solution. The performance of the actuator is studied in terms of maximum bending and actuation rate compared with a solid, uniformly 3D printed, and topology optimized actuator. The experimental results proved the effectiveness of $\mathrm{TO}$ on achieving higher bending deformation and actuation rate against a uniformly 3D printed actuator.
\end{abstract}

Keywords: Topology optimization, 4D printing, 3D printing, Soft actuator

\section{Introduction}

Until recently, production of parts in robotics such as actuators were done by using conventional polymer extrusion techniques and machining individual components prior to assembly $[1,2]$.

The introduction of soft robotics and digital additive manufacturing changed the production of robots with capabilities of delivering delicate tasks through their compliant material nature and intrigued shapes [3]. 
The rapid advancements in the emerging field of soft robotics are attributed to the developments in the stimuli-responsive active materials in conjunction with the $3 \mathrm{D}$ printing techniques and innovations. These materials are a vital part of the design of soft robots as they can function in response to specific external stimuli [4].

Additive manufacturing, particularly three-dimensional (3D) printing, has drawn soft robotics researchers' attention as it does not require molding and post-processing operations. Introducing smart and stimuli-responsive materials to 3D printing of soft robot actuators resulted in a different paradigm, known as four-dimensional (4D) printing as an elaborate form of the 3D printed soft actuator. In other words, 4D printing literally refers to the 3D printed soft robot actuator showing spatial motion over time as its fourth dimension in response to external stimuli. In addition to the manufacturing advantages, 4D printing can also be used as a powerful tool for complementing the optimal functionality of soft actuators and soft robots by facilitating rapid manufacturing [1, 5-9].

3D printing builds up material layer by layer, enabling practicality of the geometric complexity arising from topology optimization (TO) in soft robotics. Because of the layered manufacturing process, it is unnecessary to constrain the design to the solid infill when designing mechanical components; instead, the porous infill can be a good alternative, which demonstrates key advantages over the solid infill in enhancing the functionalities, such as the maximum bending. The majority of 3D printing design of such devices is based on trial and error, which can be a satisfactory methodology when dealing with rigid structures showing linear response. However, the often-unpredictable non-linear response of soft actuators makes the design process more time-consuming and costly. Yet, the finite-element analysis (FEA) tools can be employed, combined with TO as a way for predicting the behavior of soft actuators prior to fabrication. The optimization engines incorporate FEA for modeling and evaluating the current performance of geometric model in terms of pre-defined objectives in one run so as to reduce time and labor costs in the design phase.

In a recent study, hydrogels and shape memory plastics were used to demonstrate the mechanical response to targeted radiation [10]. Some of the mechanical properties of hydrogels such as the reversible dilatational strain of up to ten times the original volume can be utilized in soft robotic applications [11, 12]. Porous hydrogels can be 3D printed into structures to mimic natural muscles [13]. Reversible mechanical movement in response to electrical input was demonstrated by hydrogels in an electrochemical cell $[4,14]$. The 
hydrogel electro-actuation is induced by the osmotic pressure, created by the concentration gradient of mobile ions between the gel and the solution [15]. Diffusion of ionic species through the membrane is the key for reversible movement of electroactive polymer actuators [16].

This research studies porosity optimization of the electrically controllable 3D printed soft actuator and its effectiveness on actuation performance, particularly the maximum bending of actuator endpoint. TO and FEA are incorporated to explore different possibilities of increasing the porosity, and in turn the bending of soft actuator while keeping constant volume fraction. This paves the way for the design and development of a large variety of optimized soft actuators.

\section{Topology Optimization of 3D Printed Soft Actuator}

The TO methods are developed based on exploring the optimal structure by removing the materials from unfavorable areas and placing them in favorable areas of the structure. The soft kill option (SKO) method is a representative of this optimization type, where materials are applied in places of high stress, and removed from the low-stress areas. This method results in a porous structure with different material densities. Notable TO methods, such as the solid isotropic material with penalization (SIMP) method [17], the bi-directional evolutionary structural optimization (BESO) [18], and the level-set method [19] have been principally developed to identify the stiffest structure (through compliance minimization) for given mass and volume constraints. For instance, in the soft-kill BESO method, only the values of the normalized density of 1 and $\rho$ min are permitted, with $\rho$ min describing the minimum normalized density and thus the minimum material value, so that no singular matrices are created [20].

\subsection{Structure of the Optimization Problem}

The 3D printed soft actuator in this study is driven by the osmotic pressure. The bending of polyelectrolyte hydrogel actuator is justified by the Donnan effect. This means that the motion of counterions initiated by the applied voltage leads to the ionic gradient within the hydrogel networks along the direction of the electric field. This results in an osmotic pressure difference within the hydrogel structure, and consequently causes the deflection of 3D printed actuator toward the counter electrode. Several factors can be considered to characterize the behavior of the actuator, such as the effects of the ionic strength of electrolyte solution and 
input voltage on the bending behavior of the 3D printed polyelectrolyte actuator [10]. The optimum ionic strength of electrolyte to achieve the maximum bending angle of the 3D printed actuator was achieved [3]. It was also reported that different patterns of 3D printed actuators showed different bending deflections. Although it was reported that mainly due to the porosity made by the 3D printer, the $3 \mathrm{D}$ printed actuator of uniform lattice was more pliable in comparison with the solid cast actuator [3], there still exist more areas for optimizing the $3 \mathrm{D}$ printed pattern to attain the utmost bending with the same amount of material.

In this paper, a soft actuator is developed based on TO, in which instead of starting from a predefined uniform lattice, the exploration starts from a dense non-porous solid model of material. Distributed loading and boundary conditions are defined based on the specifications of the actuator as a cantilever beam under distributed force that reflects the osmotic pressure. Our main objective here is porosity which is directly correlated to actuator performance. With preserving the volume fraction and stiffness while increasing the number of layers in the 3D printed actuator, we indeed manipulate porosity with optimizing the arrangement of 3D printed strand layers which leads to more bending or more displacement of the actuator endpoint.

The loading and boundary conditions of a 3D printed polyelectrolyte actuator are shown in Fig. 1, where the actuator is fixed at one end and subjected to a uniform pressure along its length. Therefore, the soft actuator is designed to maximize its bending deformation to reach its full bending capacity. Using a stress-based topology optimization results in a large number of constraints for the optimization because stress is a local quantity [21]. Therefore, the computational complexity increases. In addition, stress can be highly nonlinear with respect to the design variable, which can cause additional convergence problems during the optimization [21]. We thus use a stiffness-based optimization, although the results could be a little bit worse than applying the stress-based concept.

The TO problem for a soft actuator is modeled based on the SIMP method, with the design goal being set as the minimum strain energy or maximum stiffness of the actuator. So only the necessary areas should be provided with material. A volume constraint is also introduced to regulate the stiffness of the soft actuator and guarantee general convergence of the optimization problem [22]. 


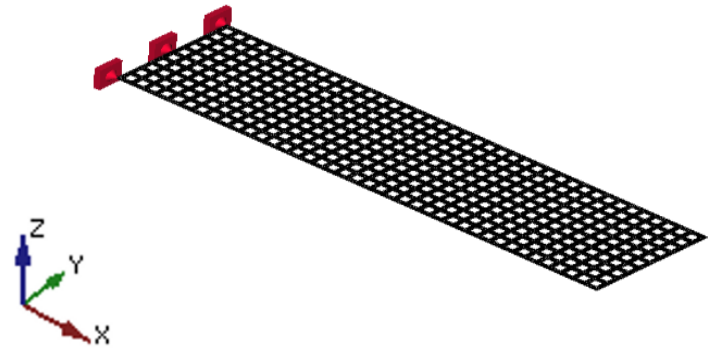

(a)

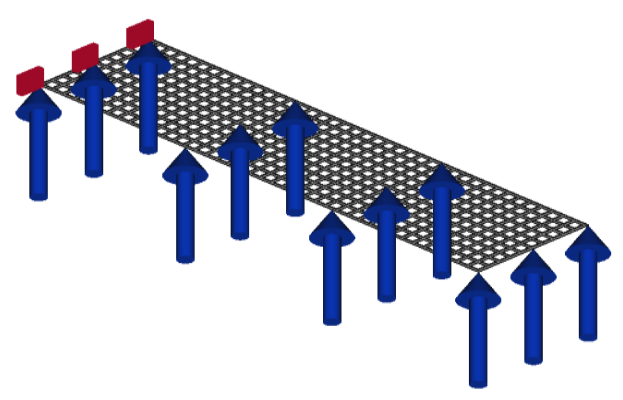

(b)

Fig. 1. Uniformly 3D printed lattice actuator (a) boundary constraint; (b) applied forces

\subsection{Determination of Sensitivity}

Several objectives can be used to determine the sensitivity. Depending on the application, direct objectives such as stress, strain and displacement can be used [23, 24]. The most common objective in TO is the change in strain compliance $\Pi_{\text {Strain }}=\frac{1}{2} \boldsymbol{u}^{T} \boldsymbol{K} \boldsymbol{u}$ of the original model with respect to the improved variant. With each iteration step, two system responses are calculated from a model with solid material and distributed material, whose combination is used for the statement of sensitivity [25]. The element's strain energy can be used when the size of element has no influence on the local strain energy. By normalizing the density with respect to the local element volume, the strain energy density $\widehat{\Pi_{o b}}$ for each element $e$ can be used as a suitable objective function for the optimization of unevenly structured meshes as:

$\widehat{\Pi_{o b j, e}}=\frac{\partial \Pi_{\text {Strain }}}{\partial \Omega}=\frac{1}{2} \frac{\partial \boldsymbol{u}^{T} \boldsymbol{K u}}{\partial \Omega}$

where $\boldsymbol{u}$ is the node displacement vector, and $\boldsymbol{K}$ is the stiffness matrices over the design field of $\Omega$. The design domain $\Omega$ is discretized into $N$ elements, the elementwise density $\rho_{e}$ is taken as a design variable continuously defined between 0 and 1 . For a single element, its Young's modulus $E_{e}$ is described by a power-law interpolation of the solid material phase $E$ and the void phase $E_{\min }$, which is given by [26]: 
$E_{e}\left(\rho_{e}\right)=E_{\text {min }}+\left(E_{0}-E_{\text {min }}\right) \rho_{e}^{p}, e=1,2, \ldots, N$

The elementwise stiffness matrix $\boldsymbol{K}_{e}$ is then obtained from the following formula as:

$\boldsymbol{K}_{e}=\boldsymbol{K}_{0, e} E_{e}\left(\rho_{e}\right)=\boldsymbol{K}_{0, e}\left(E_{\min }+\left(E_{0}-E_{\min }\right) \rho_{e}^{p}\right), e=1,2, \ldots, N$

where $\boldsymbol{K}_{0, e}$ is the elementwise stiffness matrix with a unit Young's modulus for solid material.

The energy density in a discrete finite element, with volume $V_{e}$ and node displacement $\boldsymbol{u}_{e}$ for each element, can be calculated as:

$\widehat{\Pi_{o b_{\jmath}, e}}=\frac{1}{2} \frac{\boldsymbol{u}_{e}^{T} \boldsymbol{K}_{e} \boldsymbol{u}_{e}}{V_{e}}$

The system's energy density can thus be directly used to describe the local system properties while incorporating different element types. By differentiating the element energy with respect to the design variable $\rho_{e}$, the normalized element density of the material, the suitable sensitivity for a single element $e$ can be obtained as $[25,27,28]$ :

$\frac{\partial \Pi_{\partial b_{j}, e}}{\partial \rho_{e}}=\frac{1}{2 V_{e}}\left(\frac{\partial \boldsymbol{u}_{e}^{T}}{\partial \rho_{e}} \boldsymbol{K}_{e} \boldsymbol{u}_{e}+\boldsymbol{u}_{e}^{T} \frac{\partial \boldsymbol{K}_{e}}{\partial \rho_{e}} \boldsymbol{u}_{e}+\boldsymbol{u}_{e}^{T} \boldsymbol{K}_{e} \frac{\partial \boldsymbol{u}_{e}}{\partial \rho_{e}}\right)$

Considering the derivative of the system equation $\boldsymbol{K} \boldsymbol{u}=\boldsymbol{f}$ with respect to the design variable, the following applies to a single element:

$\boldsymbol{K}_{e} \frac{\partial \boldsymbol{u}_{e}}{\partial \rho_{e}}+\frac{\partial \boldsymbol{K}_{e}}{\partial \rho_{e}} \boldsymbol{u}_{e}=\frac{\partial \boldsymbol{f}_{e}}{\partial \rho_{e}}$

Since the external load is independent of the density, Eq. (6) yields:

$\frac{\partial \boldsymbol{K}_{e}}{\partial \rho_{e}} \boldsymbol{u}_{e}+\boldsymbol{K}_{e} \frac{\partial \boldsymbol{u}_{e}}{\partial \rho_{e}}=0$

Accordingly, Eq. (7) can be rearranged to:

$\boldsymbol{K}_{e} \frac{\partial \boldsymbol{u}_{e}}{\partial \rho_{e}}=-\frac{\partial \boldsymbol{K}_{e}}{\partial \rho_{e}} \boldsymbol{u}_{e}$

Transposing Eq. (8) leads to:

$$
\left(\boldsymbol{K}_{e} \frac{\partial \boldsymbol{u}_{e}}{\partial \rho_{e}}\right)^{T}=\left(-\frac{\partial \boldsymbol{K}_{e}}{\partial \rho_{e}} \boldsymbol{u}_{e}\right)^{T}
$$


Through the symmetric stiffness matrix, we can rewrite Eq. (9) as:

$$
\frac{\partial \boldsymbol{u}_{e}^{T}}{\partial \rho_{e}} \boldsymbol{K}_{e}=-\boldsymbol{u}_{e}^{T} \frac{\partial \boldsymbol{K}_{e}}{\partial \rho_{e}}
$$

By substituting Eqs. (8) and (10) into Eq. (5), the sensitivity of the strain energy density can be obtained as:

$\frac{\partial \Pi_{o b_{j}, e}}{\partial \rho_{e}}=\frac{1}{2 V_{e}}\left(-\boldsymbol{u}_{e}^{T} \frac{\partial \boldsymbol{K}_{e}}{\partial \rho_{e}} \boldsymbol{u}_{e}+\boldsymbol{u}_{e}^{T} \frac{\partial \boldsymbol{K}_{e}}{\partial \rho_{e}} \boldsymbol{u}_{e}-\boldsymbol{u}_{e}^{T} \frac{\partial \boldsymbol{K}_{e}}{\partial \rho_{e}} \boldsymbol{u}_{e}\right)$

which is simplified as

$\frac{\Pi_{O b_{j}, e}}{\partial \rho_{e}}=-\frac{1}{2 V_{e}} \boldsymbol{u}_{e}^{T} \frac{\partial \boldsymbol{K}_{e}}{\partial \rho_{e}} \boldsymbol{u}_{e}$

The differentiation of the modified stiffness matrix in Eq. (3) leads to:

$\frac{\partial \boldsymbol{K}_{e}}{\partial \rho_{e}}=\boldsymbol{K}_{0, e}\left(E_{0}-E_{\min }\right) p \rho_{e}^{p-1}$

Then, the sensitivity can be directly determined by substituting Eq. (13) into Eq. (12) as:

$\frac{\Pi_{\widehat{O b J}, e}}{\partial \rho_{e}}=-\frac{1}{2 V_{e}}\left(E_{0}-E_{\min }\right) p \rho_{e}^{p-1} \boldsymbol{u}_{e}^{T} \boldsymbol{K}_{0, e} \boldsymbol{u}_{e}$

where $u_{e}$ results from solving the system of equations with a distributed density and material characteristic values $[23,25]$. Using the sensitivity of Eq. (14), the influence of every single change in the element itself can be investigated $[4,7]$.

\subsection{Sensitivity Filtering}

In practice, it has been shown that a direct use of the selected sensitivities leads to a checkerboard structure [24]. A compensation for this effect can be achieved with higherorder elements, i.e. quadratic or cubic elements, which usually take much longer to calculate [24]. The necessity of filtering is shown in Fig. 2. The marked area shows a pixel-like solution structure with no filtering $\left(r_{\min }=1\right)$. To prevent this, the filter limit has been increased to the boundary of $r_{\min }=3$ or $r_{\min }=5$ to remove the checkerboard-like structure and smoother contours. 


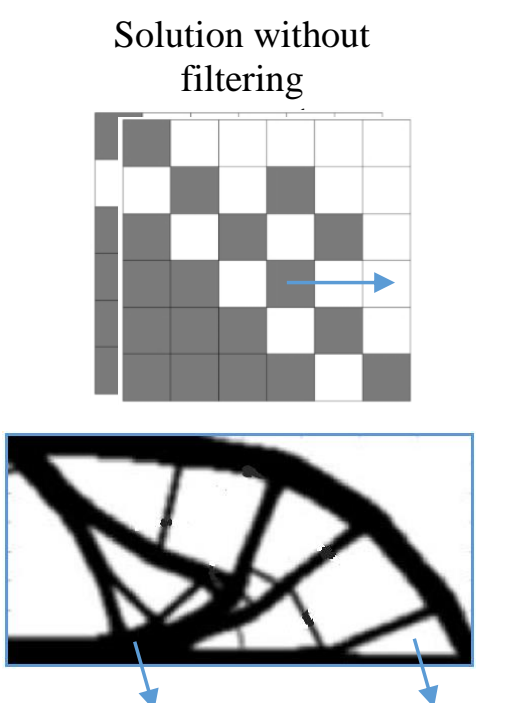

Full material
Rectangular filter

$$
r_{\min }=3
$$
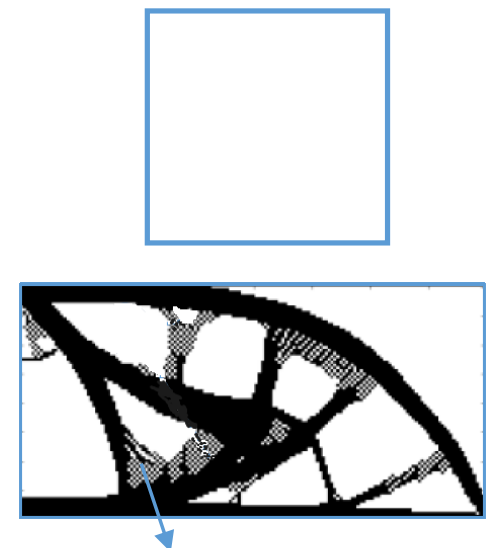

Checkerboard
Filtered solution
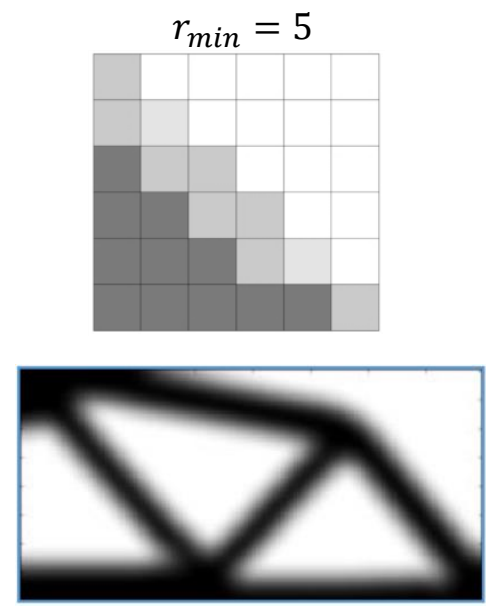

Fig. 2. Checkerboard pattern filtering

The principle of filtering is inspired by the methods from digital image processing. This results in averaging of the sensitivities and densities of the solution structure, and leads to blurred solution contours without checkerboard patterns. To be able to use these filter strategies, the conditions of the pixel structure of a digital image must be adapted for the finite element mesh. This allows the results and methods developed in image processing to be transferred directly to TO. A filtering of the signal $\left(\frac{\Pi \widehat{O b_{J}, e}}{\partial \rho_{e}}\right.$ or $\left.\rho_{e}\right)$ of the element $e$ takes place via a so called filter mask $\widehat{\boldsymbol{H}}_{e}$. Figure 3 shows a simple example of density filtering in some area elements. The adjacent elements in the filter area, $\boldsymbol{\Omega}_{F}$, are sorted into a matrix. The matrix elements are then multiplied separately, and their summation results in a new filtered value $[29,30]$.

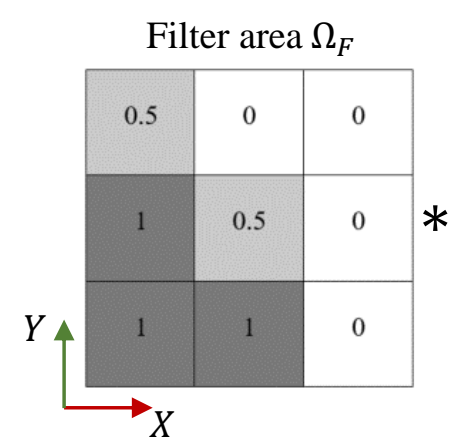

Filter mask $\widehat{H}_{e}$

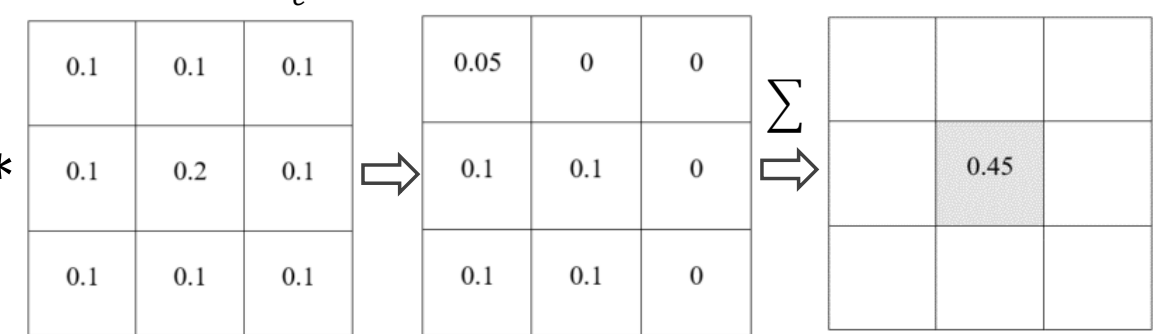

Fig. 3. Example of the filter strategy 
The TO algorithm used in this study is illustrated in Fig. 4. The SIMP method is adopted to solve the TO problem, where densities of the discretized elements are taken as design variables [31]. The SIMP method and a Euclidian distance filter matrix are used for smoothing the sensitivity result and avoiding checkboard results [32, 33]. The loading and boundary conditions of the 3D printed soft actuator are modeled as constraints of the optimization model. Thereafter, the optimization problem is solved iteratively with the guidance of sensitivity information. To ensure the minimum structural stiffness and convergence of the optimization problem, volume constraint is also introduced [34]. In addition, a standard method of moving asymptotes (MMA) is used to comply with the volume restriction in the TO problem. By doing so, we improve the optimal performance of 3D printed actuator, which is the maximum bending angle in this study, through the optimization of printed strands on top of each layer.

The optimization results in terms of objective function and volume fraction over iteration history are provided in Fig. 5. The figure illustrates that the objective function decreases gradually and reaches its minimum as the volume fraction is satisfied, with around 39 iterations. The corresponding optimal solutions for different layers of soft actuator are shown in Fig. 6, where the optimized layers are developed with the same amount of materials as the uniformly distributed $3 \mathrm{D}$ printed soft actuator.

\section{3D Printing of the Soft Actuator}

The key issue in fabrication of soft actuator using 3D printer is solidification of hydrogel strand upon extrusion from the printer nozzle. The preparation of hydrogels used for printing which can retain their structural integrity after extrusion has been studied through several approaches based on the types of hydrogel materials and their subsequent applications [3537]. One primary way is to use the solvents of high molecular weight, which result in polymer inks with high viscosity. Cross-linkers (e.g., ultraviolet (UV) or forced air drying) can also be utilized in the solidification of hydrogels after printing or adjusting the print platform temperature to accelerate the solidification process. Solvent casting is another method that works based on the solidification of printed filament through solvent evaporation [38]. The wet-spinning method based on solvent exchange in a bath of cross-linker has also been used for 3D printing of hydrogels (e.g. EtOH) [39]. 
In this study, the chitosan hydrogel was 3D printed by the liquid hardening method [40] with an EnvisionTEC GmbH bioplotter. The computer-aided design (CAD) model of the actuator was created in Solidworks and subsequently loaded into the bioplotter. $1.6 \mathrm{~g}$ of chitosan of medium molecular weight (SigmaAlrich, Australia) was added into $0.8 \mathrm{ml}$ of $1.0 \% \mathrm{v} / \mathrm{v}$ acetic acid solution and stirred for $2 \mathrm{~h}$, followed by centrifugation. The resulting extrudate was poured into the syringe of the bioplotter. Solidification of the extrudate took place in a 0.25 M sodium hydroxide solution. The topology-optimized actuators were printed in uniform layers (Figs. 6 and 7). The 3D printer settings and experimental details for printing chitosan are provided in an earlier publication of the authors [3].

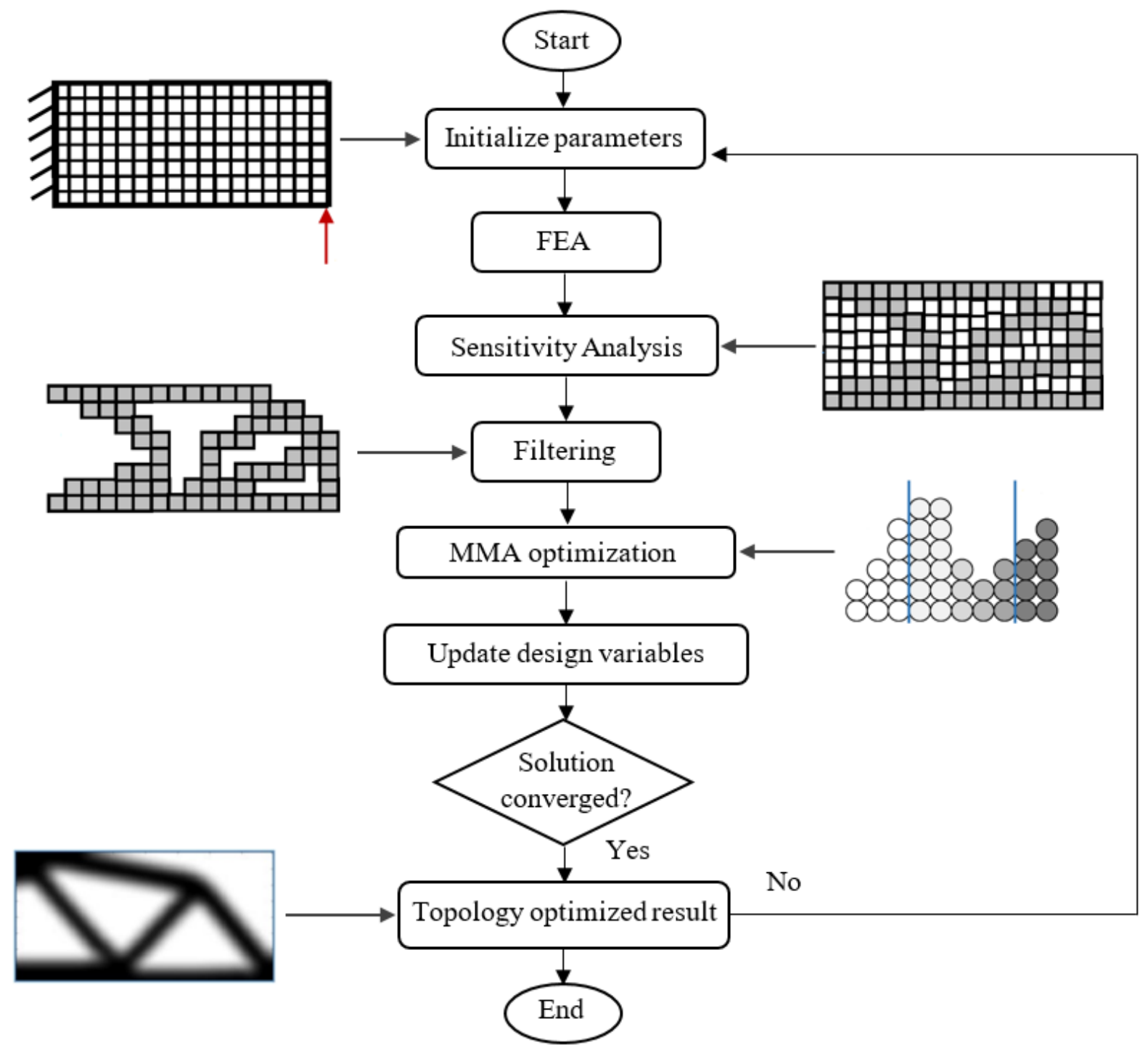

Fig. 4. Flowchart of the TO algorithm used in this study 


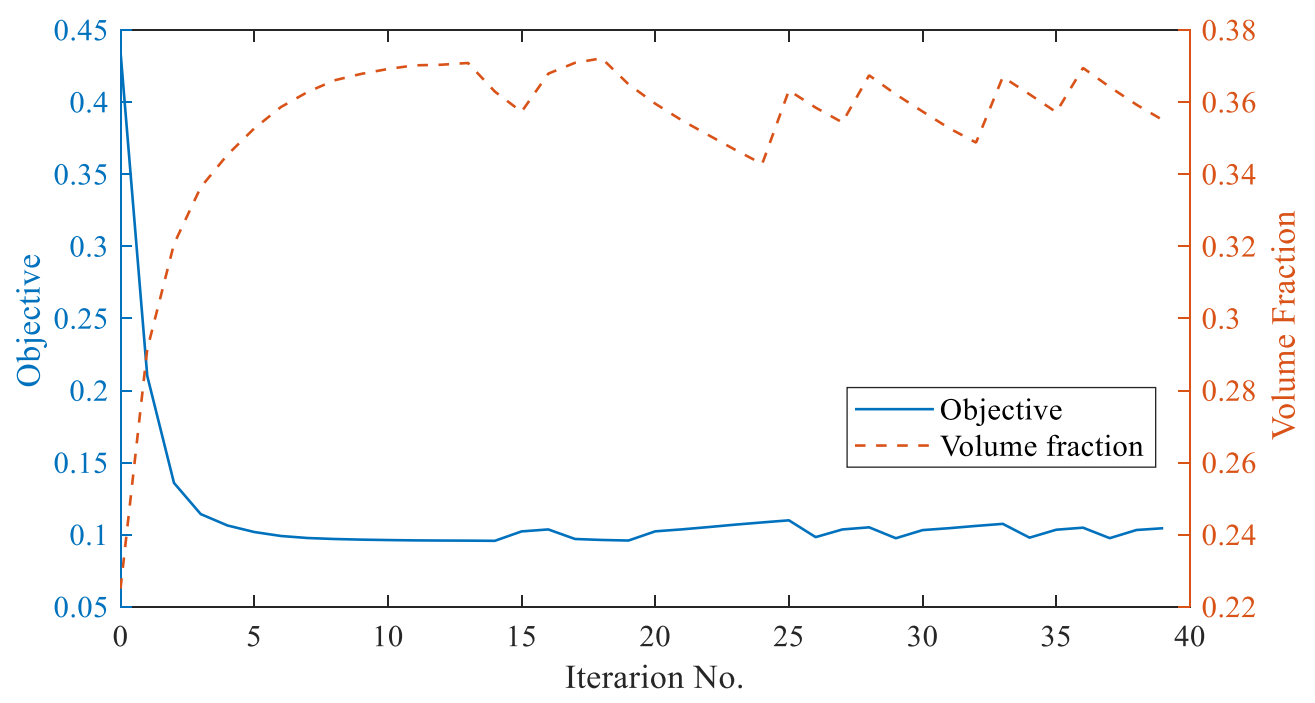

(a)

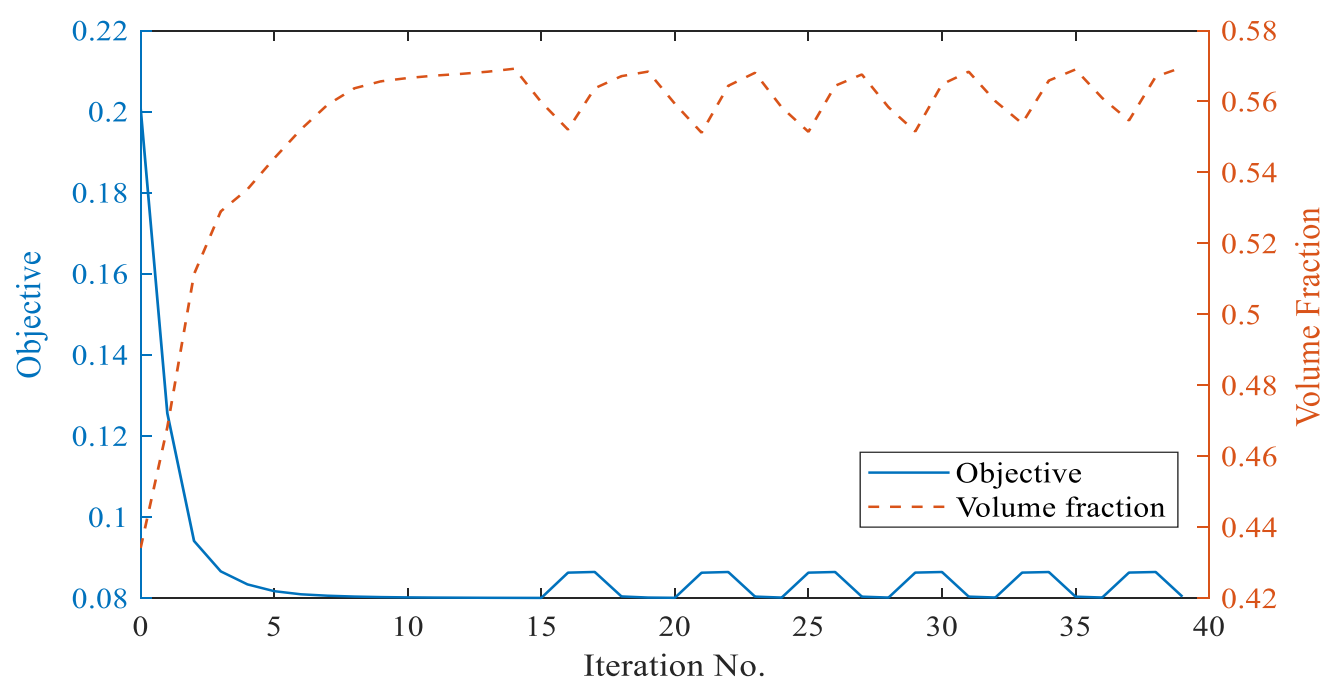

(b)

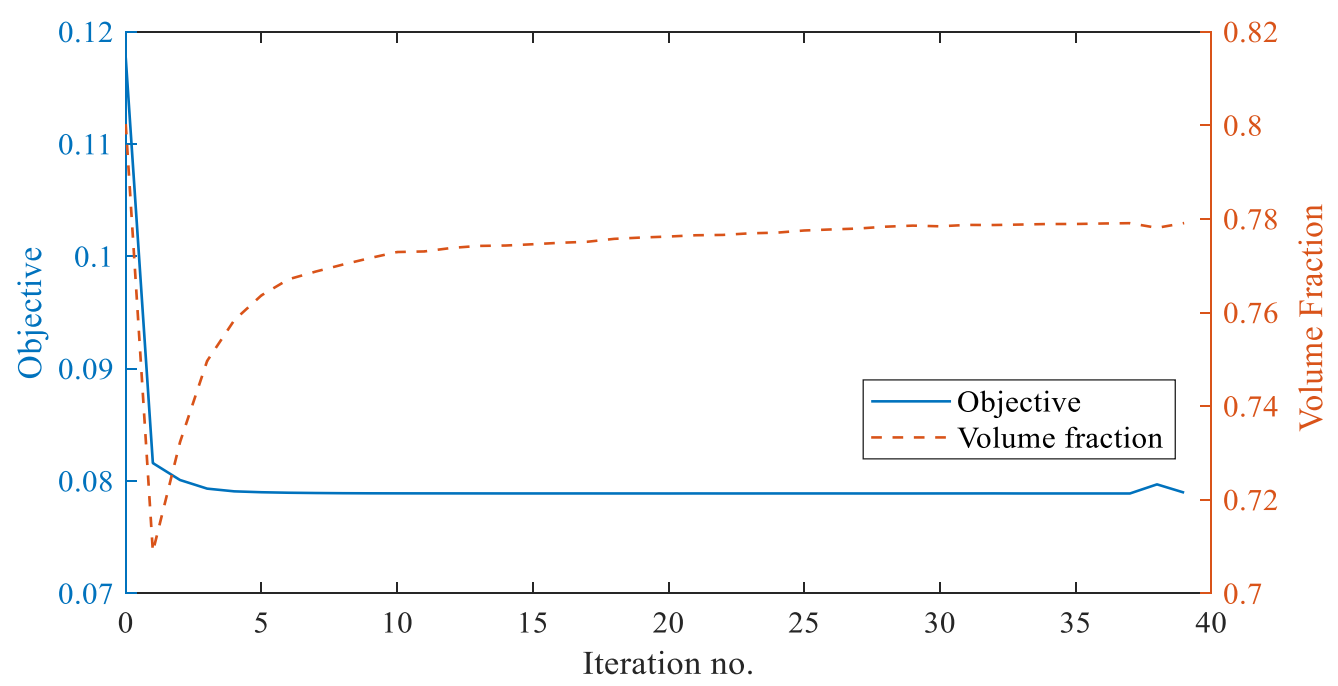

(c) 
Fig. 5. TO results versus iterations with $p=2$ (a) $v=0.4$; (b) $v=0.6$; and (c) $v=0.8$
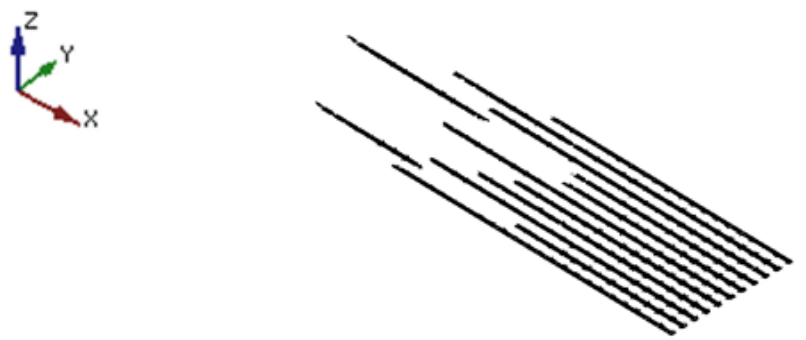

Layer 5

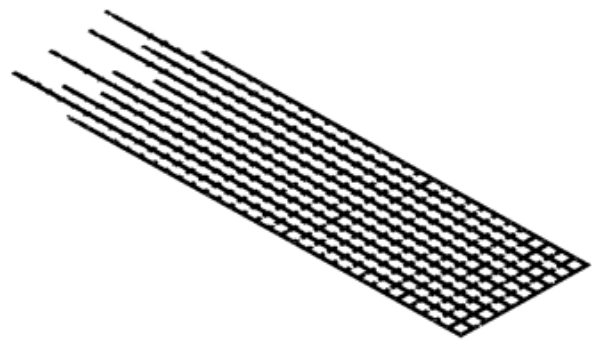

Layers $3 \& 4$

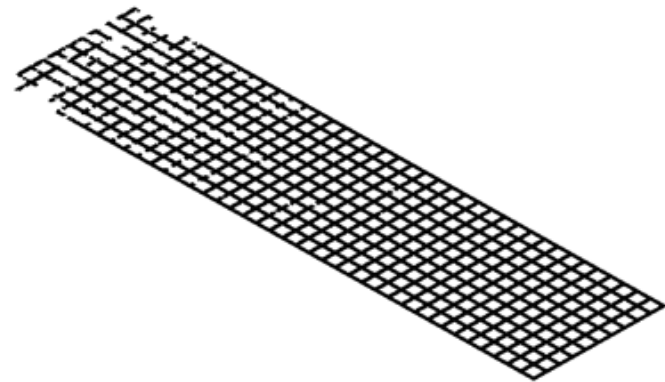

Layers $1 \& 2$

Fig. 6. The topology-optimized 3D printed actuator layers (the odd and even layers are printed in the $X$ and $Y$ directions, respectively)

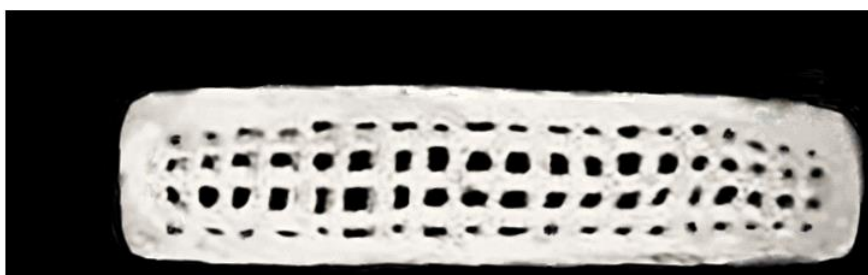

(a)

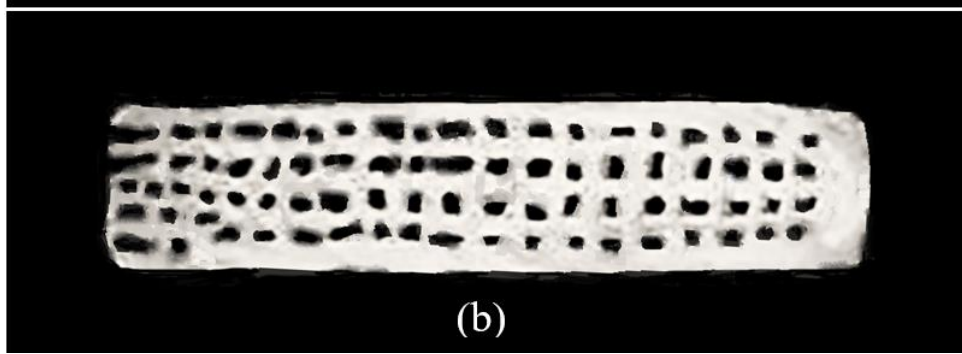

Fig. 7. 3D printed soft actuators: (a) with uniform lattice; and (b) topology-optimized 3D printed actuators 


\section{Experimental Results and Discussions}

To assess and show the effectiveness of the proposed TO design in fabrication of 3D printed soft actuator, a range of experiments were conducted to measure the free bending trajectories of the actuators' endpoints. The 3D printed soft actuator is fixed on the supportive end using a paper clipper, leaving the endpoint deform freely in the horizontal plane. An osmotic pressure is exerted on the surface of the $3 \mathrm{D}$ printed soft actuator through the input voltage signal applied on two steel mesh electrodes.

First, the actuators were tested with three different input signals of $3 \mathrm{~V}, 5 \mathrm{~V}$, and $7 \mathrm{~V}$. All the experiments results are repeated three times and the averaged results are depicted in the following figures. The experiments results shown in Figs. 8 and 9 prove that the topologyoptimized 3D printed soft actuator underwent larger endpoint displacement compared with the $3 \mathrm{D}$ printed actuator with uniform lattice. Also, it can be found from the details of the actuators' bending in Fig. 8 that not only the bending performance of the topology-optimized $3 \mathrm{D}$ printed actuator outperformed the 3D printed actuator with uniform lattice when the input signal is on, up to $50 \mathrm{~s}$ in the graphs, but also the free bending relaxation of the topologyoptimized 3D printed actuator occurred at a higher rate when the input signal was off, after $50 \mathrm{~s}$ in the graphs. That could be the main reason to justify the overlap of the endpoint locations for both the $3 \mathrm{D}$ printed actuator with uniform lattice and the TO 3D printed soft actuator at the end of the actuation cycle, while the maximum bending was achieved by the TO 3D printed soft actuator during the actuation. 
(a)
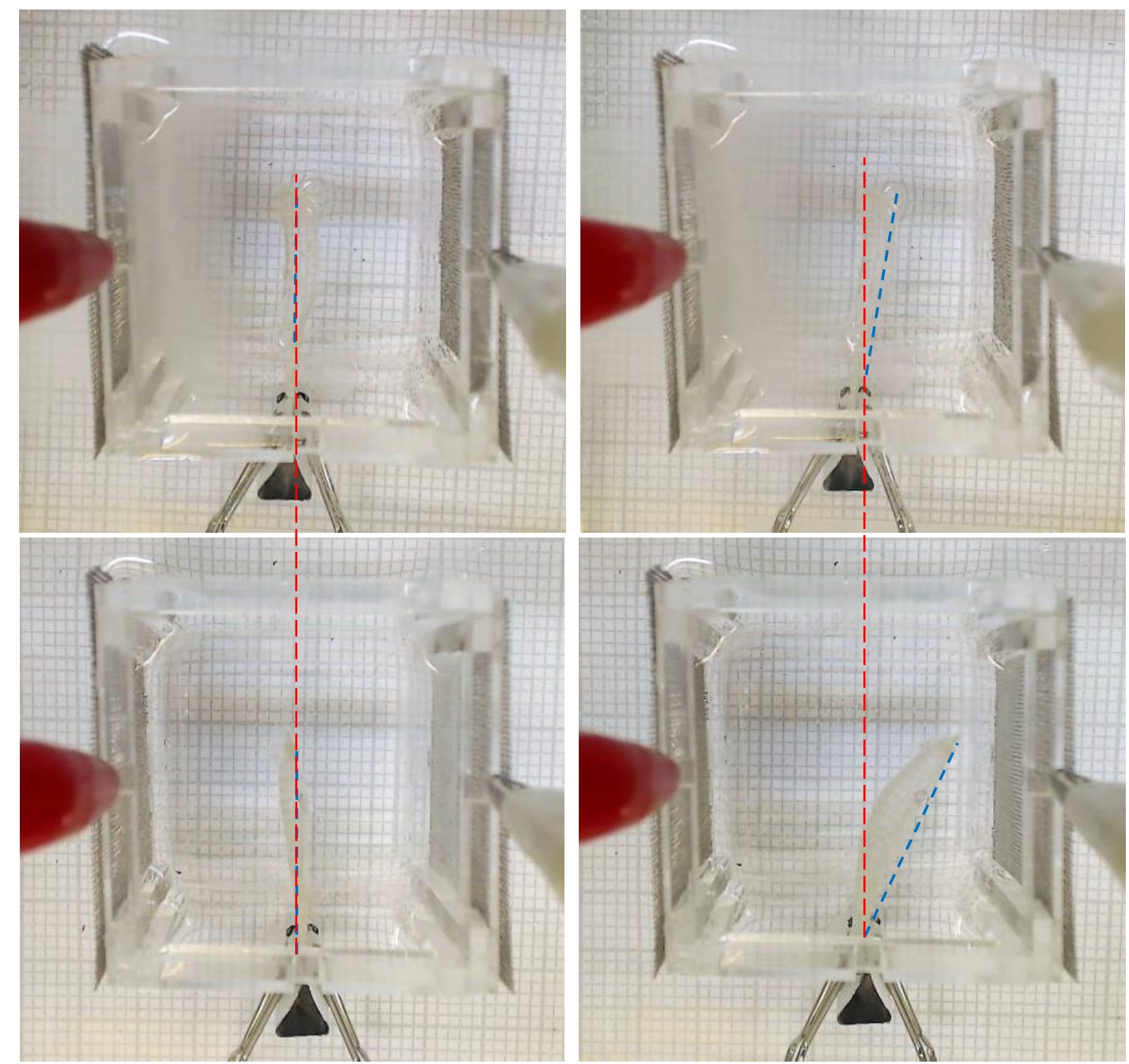

Fig. 8. 3D printed actuators' end-point displacements over time (a) 3D printed lattice actuator; (b) topology-optimized $3 \mathrm{D}$ printed actuator

Additionally, hysteretic tests were conducted on limited on-off cycles of input signal. The results of hysteretic experiments in response to the input signal of $5 \mathrm{~V}$, depicted in Fig. 10, reveal that the functionality of actuator gradually plunges with repeating the cycles in free bending trajectory. The hysteretic deviation from the maximum endpoint displacements is indicated by peak envelop lines in Fig. 10. Also, when comparing between different cycles, large deviation up to $30 \%$ is observed for the topology-optimized 3D printed soft actuator. 


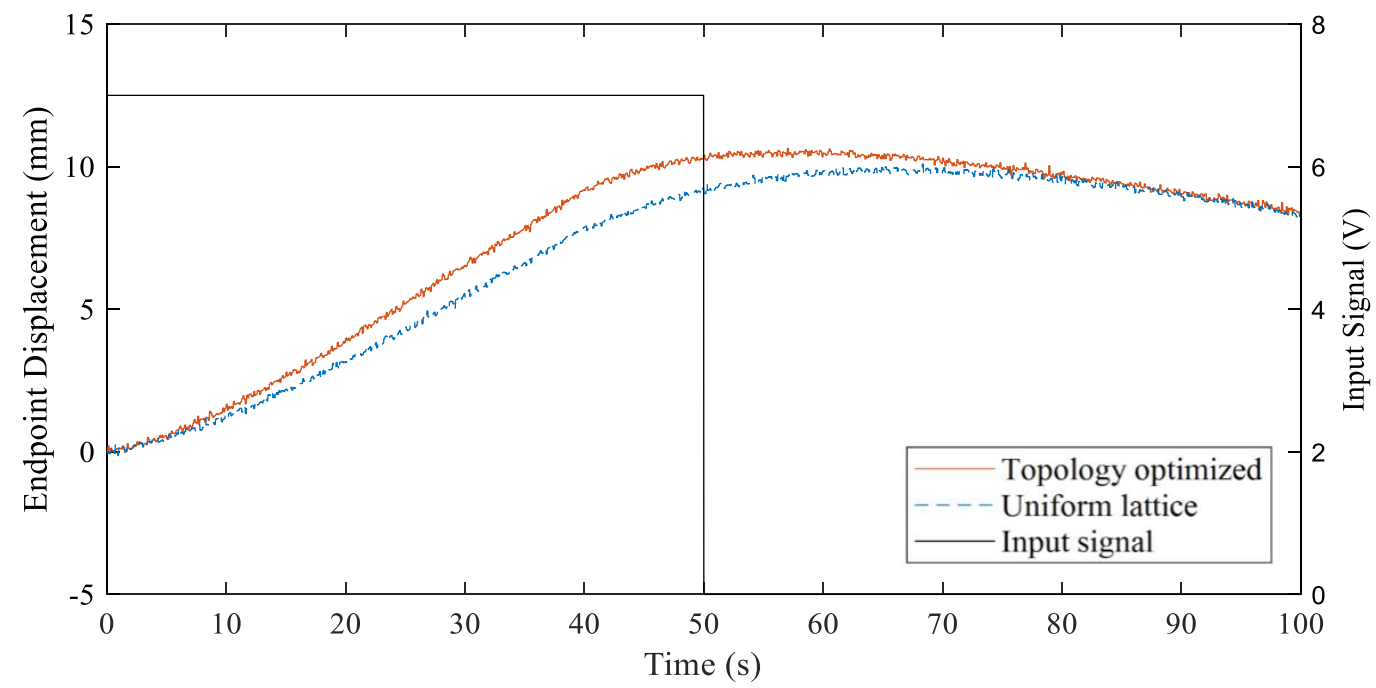

(a)

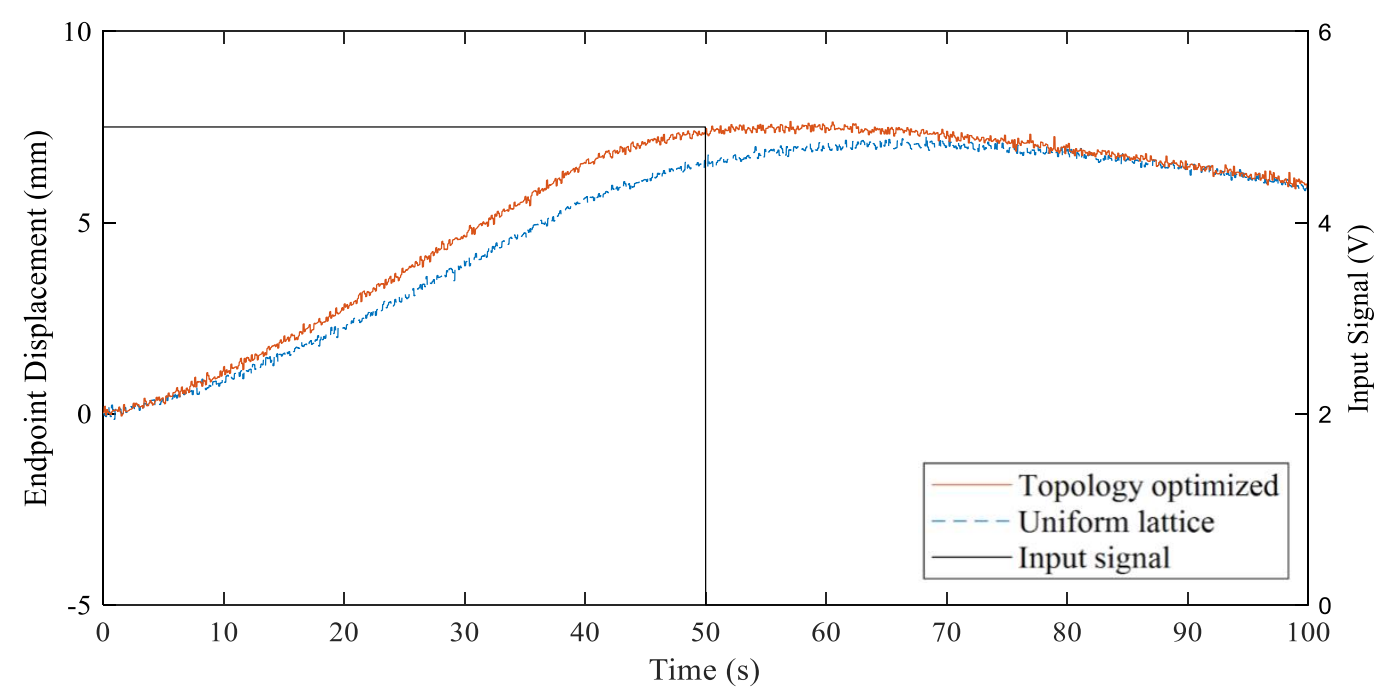

(b)

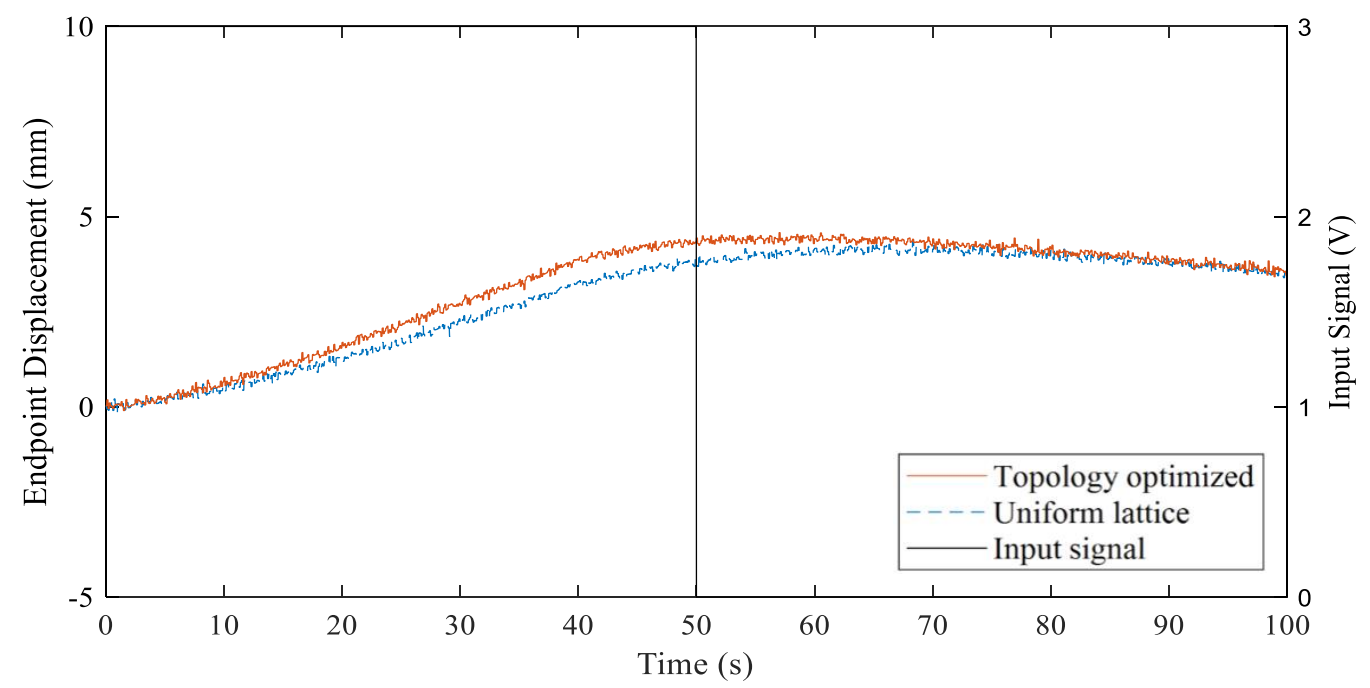

(c)

Fig. 9. Free-bending trajectory of 3D printed soft actuators under different input signals; (a) 7 $V$; (b) $5 V$; (c) $3 V$ 


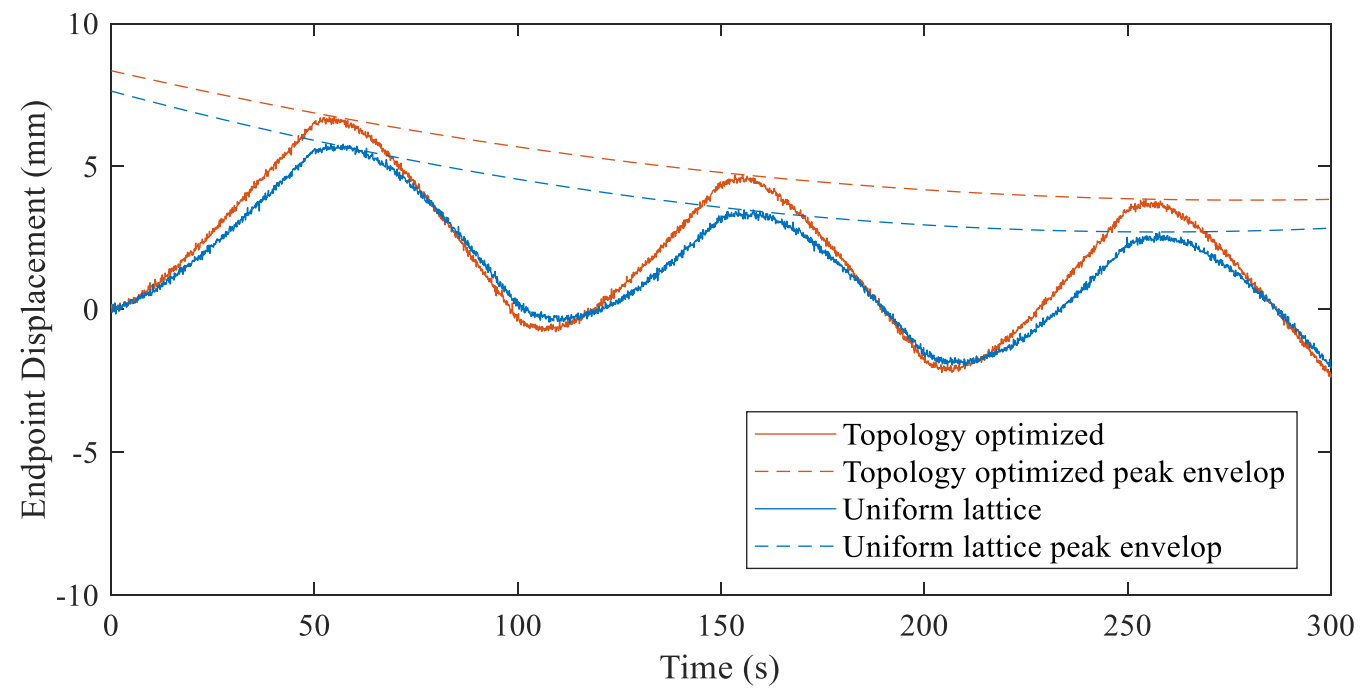

(a)

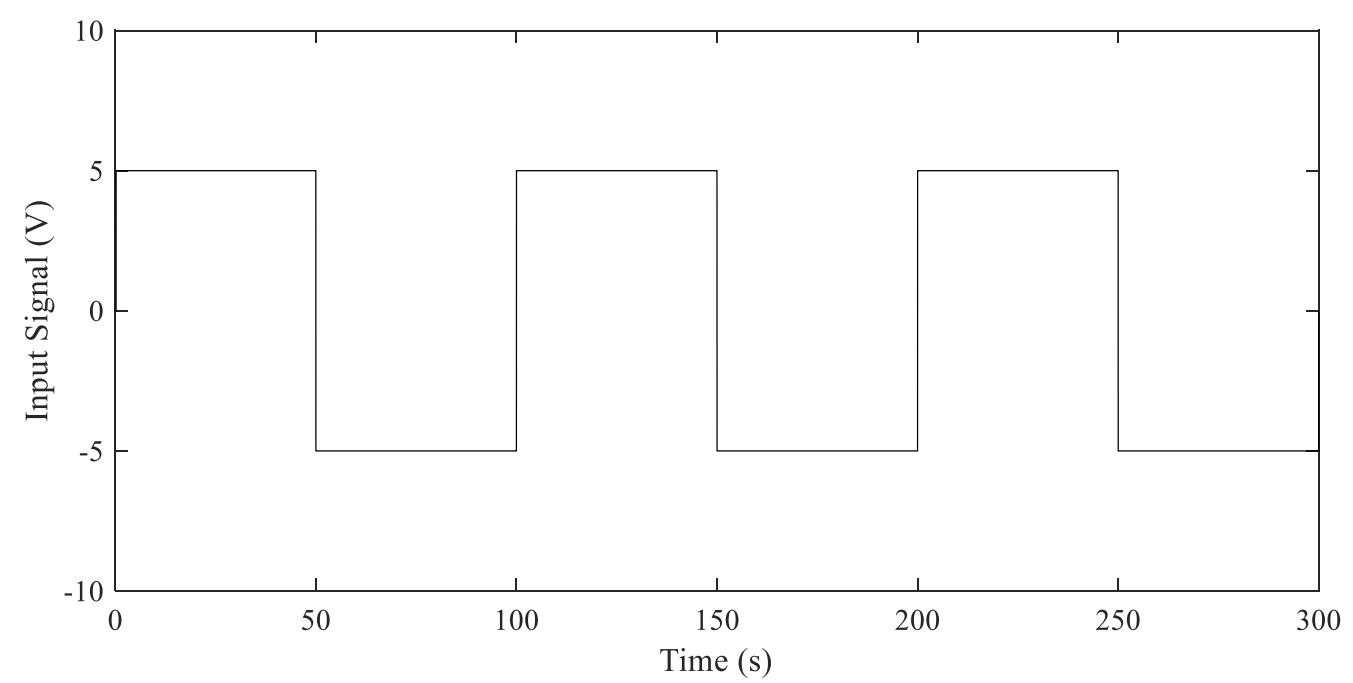

(b)

Fig. 10. (a) Hysteretic test results of 3D printed soft actuators under (b) three on-off cycles of input signal of $5 \mathrm{~V}$

\section{Conclusion}

In this study, 4D printing is incorporated with topology optimization to enhance the full potential of the soft actuator's functionality. The soft actuator is designed by topology optimization to achieve its maximal free bending deformation, evaluated by endpoint displacement. First, a polyelectrolyte soft actuator is 3D printed using a bioprinter and a polyelectrolyte hydrogel. The topology-optimized 3D printed soft actuator is then searched according to the stiffness requirements by engineering the material arrangement within the considered volume. The experiments showed that, due to porosity rearrangement within the constant volume of 3D printed material, the topology-optimized 3D printed soft actuator 
achieved more functionality compared with the 3D printed actuator with uniform lattice. The results imply the power of topology-optimization-based design approach for leveraging full potential of the design space of high-performance 3D printed soft robots and actuators. For further investigations, a stress-based concept for topology optimization can be used to increase the deflection of actuator regarding a maximum-stiffness-based design that is not always equivalent to a minimum-stress-based design.

\section{References}

1. Cohen, E., et al. Design Methodologies for Soft-Material Robots Through Additive Manufacturing, From Prototyping to Locomotion. in ASME 2015 International Design Engineering Technical Conferences and Computers and Information in Engineering Conference. 2015. American Society of Mechanical Engineers.

2. Koloor, S., et al., FE model-based construction and progressive damage processes of FRP composite laminates with different manufacturing processes. International Journal of Mechanical Sciences, 2018. 141: p. 223-235.

3. Zolfagharian, A., et al., Polyelectrolyte Soft Actuators: 3D Printed Chitosan and Cast Gelatin. J 3D Printing Additive Manufacturing, 2018. 5(2): p. 138-150.

4. Zolfagharian, A., et al., Evolution of 3D printed soft actuators. J Sensors Actuators A: Physical, 2016. 250: p. 258-272.

5. Zolfagharian, A., et al., Pattern-driven 4D printing. J Sensors Actuators A: Physical, 2018. 274: p. 231-243.

6. Bodaghi, M., W.-H.J.S.M. Liao, and Structures, 4D Printed Tunable Mechanical Metamaterials with Shape Memory Operations. 2019.

7. Bodaghi, M., A. Damanpack, and W. Liao, Self-expanding/shrinking structures by $4 D$ printing. J Smart Materials Structures, 2016. 25(10): p. 105034.

8. Bodaghi, M., et al., 4D printing self-morphing structures. J Materials, 2019. 12(8): p. 1353.

9. Gao, B., et al., 4D Bioprinting for Biomedical Applications. Trends in Biotechnology, 2016.

10. Zolfagharian, A., et al., Rigid elements dynamics modeling of a 3D printed soft actuator. J Smart Materials Structures, 2018. 28(2): p. 025003.

11. Zolfagharian, A., et al., System Identification and Robust Tracking of a 3D Printed Soft Actuator. 2019.

12. Ionov, L., Biomimetic Hydrogel-Based Actuating Systems. Advanced Functional Materials, 2013. 23(36): p. 4555-4570.

13. O'Grady, M.L., P.-I. Kuo, and K.K. Parker, Optimization of electroactive hydrogel actuators. ACS applied materials \& interfaces, 2009. 2(2): p. 343-346.

14. Zolfagharian, A., et al., Bending control of a 3D printed polyelectrolyte soft actuator with uncertain model. J Sensors Actuators A: Physical, 2019. 288: p. 134-143.

15. Shiga, T. and T. Kurauchi, Deformation of polyelectrolyte gels under the influence of electric field. Journal of Applied Polymer Science, 1990. 39(11-12): p. 2305-2320.

16. Li, Y., et al., Electric Field Actuation of Tough Electroactive Hydrogels Cross-Linked by Functional Triblock Copolymer Micelles. ACS Applied Materials \& Interfaces, 2016. 8(39): p. 26326-26331.

17. Sigmund, O., K.J.S. Maute, and M. Optimization, Topology optimization approaches. 2013. 48(6): p. 1031-1055.

18. Huang, X., Y.J.F.E.i.A. Xie, and Design, Convergent and mesh-independent solutions for the bidirectional evolutionary structural optimization method. 2007. 43(14): p. 1039-1049. 
19. van Dijk, N.P., et al., Level-set methods for structural topology optimization: a review. 2013. 48(3): p. 437-472.

20. Bendsøe, M.P. and O. Sigmund, Material interpolation schemes in topology optimization. J Archive of applied mechanics, 1999. 69(9-10): p. 635-654.

21. Yang, R. and C. Chen, Stress-based topology optimization. J Structural optimization, 1996. 12(2-3): p. 98-105.

22. HONGYING, Z., DEVELOPMENT OF TOPOLOGY OPTIMIZED 3D PRINTED SOFT GRIPPERS AND DIELECTRIC SOFT SENSORS. 2018.

23. Schumacher, A., Mathematische Grundlagen der Optimierung, in Optimierung mechanischer Strukturen. 2013, Springer. p. 45-55.

24. Harzheim, L., Der Natur in die Karten geschaut-Optimierungsverfahren aus dem Bereich der Bionik, in Karosseriebautage Hamburg 2016. 2016, Springer. p. 3-16.

25. Huang, X. and M. Xie, Evolutionary topology optimization of continuum structures: methods and applications. 2010: John Wiley \& Sons.

26. Zhang, H., et al., Topology Optimized Multimaterial Soft Fingers for Applications on Grippers, Rehabilitation, and Artificial Hands. J IEEE/ASME Transactions on Mechatronics, 2019. 24(1): p. 120-131.

27. He, D., S.J.I.J.f.S. Liu, and M.D. Optimization, BESO method for topology optimization of structures with high efficiency of heat dissipation. 2008. 2(1): p. 43-48.

28. Kim, M.-G., J.-H. Kim, and S.-H.J.J.o.t.C.S.E.I.o.K. Cho, Topology design optimization of heat conduction problems using adjoint sensitivity analysis method. 2010. 23(6): p. 683-691.

29. Gersborg-Hansen, A., et al., Topology optimization using the finite volume method. 2005.

30. Kaup, I.A., Rekonstruktion verrauschter nichtregelmäßig abgetasteter Bilddaten. 2018.

31. Svanberg, K.J.I.j.f.n.m.i.e., The method of moving asymptotes-a new method for structural optimization. 1987. 24(2): p. 359-373.

32. Wiedemann, J., Leichtbau: Elemente und Konstruktion. 2007: Springer-Verlag.

33. Tavakoli, R., S.M.J.S. Mohseni, and M. Optimization, Alternating active-phase algorithm for multimaterial topology optimization problems: a 115-line MATLAB implementation. 2014. 49(4): p. 621-642.

34. Zhang, H., et al. Topology optimized design, fabrication and evaluation of a multimaterial soft gripper. in 2018 IEEE International Conference on Soft Robotics (RoboSoft). 2018. IEEE.

35. Stamm, C.J.I.-S., Hochgeschwindigkeitsvideoverarbeitung im Sport. 2013. 36(5): p. 431-439.

36. Wüst, S., et al., Tunable hydrogel composite with two-step processing in combination with innovative hardware upgrade for cell-based three-dimensional bioprinting. Acta biomaterialia, 2014. 10(2): p. 630-640.

37. Lee, J.M. and W.Y. Yeong, Design and Printing Strategies in 3D Bioprinting of Cell-Hydrogels: A Review. Advanced Healthcare Materials, 2016.

38. Wu, Q., et al., Solvent-cast 3D printing of chitosan hydrogel scaffolds for guided cell growth. 2016.

39. Hinton, T.J., et al., Three-dimensional printing of complex biological structures by freeform reversible embedding of suspended hydrogels. Science advances, 2015. 1(9): p. e1500758.

40. Zolfagharian, A., et al., 3D printed soft parallel actuator. Smart Materials and Structures, 2018. 27(4): p. 045019. 